\title{
Os problemas de espalhamento quântico em potenciais elementares
}

(Quantum scattering in elementary potential problems)

\author{
M.A. Cândido Ribeiro' ${ }^{1}$, V.C. Franzoni ${ }^{1}$, W.R. Passos ${ }^{1}$, E.C. Silva $^{1}$ e A.N.F. Aleixo ${ }^{2}$ \\ ${ }^{l}$ Universidade Estadual Paulista, Instituto de Biociências, Letras e Ciências Exatas, \\ Departamento de Física, São José do Rio Preto, SP, Brazil. \\ ${ }^{2}$ Universidade Federal do Rio de Janeiro, Instituto de Física, RJ, Brazil.
}

Recebido em 29/5/03; Aceito em 28/11/03

\begin{abstract}
Neste trabalho estudamos o espalhamento quântico de uma partícula por um potencial generalizado que engloba em si todas as formas de potenciais retangulares unidimensionais. Neste contexto nós calculamos as probabilidades de reflexão e transmissão de uma partícula incidente sobre este potencial generalizado e avaliamos os efeitos de sua assimetria na transmissão dessa partícula através dele para energias abaixo e acima da barreira de potencial.

Palavras-chave: espalhamento quântico, probabilidades de transmissão e reflexão, potenciais unidimensionais, efeitos de assimetria.

A potential which encompasses all kinds of rectangular one-dimensional potentials is studied in the context of quantum particle scattering. We calculate the reflection and transmission coefficients for this general potential. The effects of the potential asymmetry on transmission, at energies below and above barrier, are studied, as well. Keywords: quantum scattering, transmission and reflection coefficients, one-dimensional potentials, asymmetry effects.
\end{abstract}

\section{Introdução}

Os resultados das experiências envolvendo espalhamento e tunelamento quântico tem nos fornecido muito do conhecimento que possuímos hoje sobre a estrutura dos átomos, núcleos, bem como sobre a natureza das partículas e de suas interações [1]. No caso do espalhamento, os feixes de partículas produzidos por aceleradores de colisão ou originários de fontes radioativas são usados nas experiências como pontas de prova para o estudo da natureza dos elementos constituintes (moléculas, átomos, partículas, etc.) de alvos por eles bombardeados. Nestas experiências com espalhamento as distribuições angular e de energia junto com a intensidade do feixe de partículas espalhadas fornecem informações reveladoras relacionadas com a interação entre os elementos do alvo e as partículas do feixe incidente. Os dados obtidos destas experiências são então usados para testar as predições feitas por modelos baseados na mecânica quântica nos quais alguma forma para o potencial de interação é assumida como atuante. No caso do tunelamento quântico sabemos que ele se manifesta numa grande variedade de fenômenos nas diversas áreas da física. Alguns exemplos históricos deste fenômeno quântico são: o decaimento alfa dos núcleos em que um núcleo de $\mathrm{He}$ com energia abaixo da barreira de potencial Coulombiano tunela através dela, fenômeno que foi explicado pela teoria de Gamow, Gurney e Condon [2,3]; a emissão de campo na qual elétrons são emitidos por metais após a aplicação de um campo elétrico externo que, ao modificar a forma do

Enviar correspondência para M.A. Cândido Ribeiro. E-mail: macr@df. ibilce.unesp.br. potencial de ligação do sistema, possibilita o tunelamento de elétrons do mar de Fermi, fenômeno que foi explicado inicialmente de forma qualitativa pela teoria de FowlerNordheim [4]; os microscópios de emissão e de tunelamento onde a imagem da estrutura atômica das superfícies de certos materiais é obtida através do resultado do tunelamento por emissão de campo dos elétrons destes materiais. Outros exemplos são as reações de fusão nuclear onde os núcleos envolvidos no processo de fusão precisam tunelar através da barreira de potencial existente entre eles para que o processo se concretize; a penetração do átomo de $\mathrm{N}$ na barreira de potencial criada pelos três átomos de $\mathrm{H}$ na inversão periódica da molécula de amônia $\left(\mathrm{NH}_{3}\right)$ [5], fenômeno que foi inicialmente utilizado na fabricação de relógios atômicos; o tunelamento de elétrons em materiais semicondutores, tais como nos diodos tunel que são largamente usados nos circuitos eletrônicos rápidos devido à sua alta frequência de resposta. Nesta área da física o fenômeno de transmissão de portadores de carga através de barreiras de potencial tem uma importância muito grande [6]. Numa abordagem mais atualizada podemos citar o tunelamento ressonante de elétrons através de cavidades (quantum dots) como exemplo. Quantum dots são "átomos" fabricados artificialmente através do confinamento tridimensional de portadores de carga que simulam, assim, os elétrons aprisionados em um átomo real. Este aprisionamento ocorre na região de junção de dois ou mais materiais diferentes (heteroestruturas quânticas), tais como GaAs ou GaAlAs. Sendo assim eles apresentam propriedades similares aquelas normalmente associadas aos átomos reais, tais como estruturas de camadas, níveis quantizados de energia, etc [7-10]. O 
que os torna especiais é a possibilidade de se poder controlar seus tamanhos e suas formas através de uma tecnologia de fabricação em escala nanométrica. Esta liberdade de fabricação abre uma grande variadade de aplicações em várias áreas da física tais como na fabricação de lasers com comprimentos de onda antes inacessíveis, fabricação de chips para uma próxima geração de computadores mais rápidos, etc. Espera-se que no futuro quantum dots talvez possam tornar possível o sonho da computação quântica. Este novo campo de estudo sobre sistemas quânticos artificiais tem sido denominado de física de sistemas mesoscópicos e constitui um campo de pesquisa em grande atividade na área da física do estado sólido.

Em resumo tanto o espalhamento quanto o tunelamento quântico tem se constituido em instrumentos preciosos quando tentamos compreender a natureza e o que acontece no mundo microscópico das moléculas, átomos e partículas. Nestes estudos é rotineiro o uso de modelos que assumem para os potenciais de interação entre as partículas formas que podem ser divididas em regiões espaciais nas quais estes potenciais podem ser aproximados por patamares constantes. Neste caso, como as regiões de transição dos potenciais entre os patamares vizinhos estão restritas a regiões espaciais muito pequenas, então estas transições do potencial podem ser assumidas como abruptas. Um outro aspecto a ser ressaltado é que, a despeito da tridimensionalidade do problema do espalhamento, alguns de seus aspectos mais fundamentais podem ser apresentados e explorados em termos de modelos físicos muito simples cujos cálculos são baseados no uso de potenciais retangulares unidimensionais. De uma maneira geral, os potenciais retangulares unidimensionais oferecem um exemplo instrutivo e o seu uso no estudo do espalhamento de partículas quânticas apresenta-se sempre vantajoso como uma primeira abordagem investigativa que possibilita obter uma avaliação qualitatita rápida dos aspectos físicos mais relevantes do fenômeno.

Para estudar as propriedades ondulatórias associadas a propagação de uma partícula quântica de massa $m$ e energia assintótica $E$ que se movimenta unidimensionalmente e incide sobre um potencial $V(x)$ devemos resolver a equação de Schrödinger independente do tempo

$$
\left(-\frac{\hbar^{2}}{2 m} \frac{d^{2}}{d x^{2}}+V(x)\right) \varphi(x)=E \varphi(x)
$$

sendo a sua solução $\varphi(x)$ denominada de função de onda associada à partícula. Uma vez definida a forma do potencial $V(x)$ é possível obter a solução geral para a função de onda $\varphi(x)$ e dela identificar suas componentes associadas à incidência $\left[\varphi_{\mathrm{I}}(x)\right]$, reflexão $\left[\varphi_{\mathrm{R}}(x)\right]$ e transmissão $\left[\varphi_{\mathrm{T}}(x)\right]$ da partícula neste potencial. As densidades de correntes de probabilidades, ou fluxos, para cada uma dessas possibilidades de ocorrências podem ser obtidas através da expressão geral

$$
J(x)=\frac{\hbar}{2 m i}\left(\varphi * \frac{\partial \varphi}{\partial x}-\frac{\partial \varphi^{*}}{\partial x} \varphi\right),
$$

particularizada para cada uma das componentes da função de onda em questão. As probabilidades de reflexão $R(E)$ e transmissão $T(E)$ da partícula pelo potencial $V(x)$ são definidas como as razões dos fluxos refletido $J_{\mathrm{R}}$ e transmitido $J_{\mathrm{T}}$ para o fluxo incidente $J_{\mathrm{I}}$

$$
R(E) \equiv\left|\frac{J_{R}}{J_{I}}\right| ; \quad T(E) \equiv\left|\frac{J_{T}}{J_{I}}\right|,
$$

respectivamente. Sendo $V(x)$ um potencial real, então reflexão e transmissão são as únicas possibilidades para a partícula que incide nele, de modo que devemos ter

$$
R(E)+T(E)=1
$$

Em outras palavras, ao atingir $V(x)$, o fluxo de probabilidade incidente se desdobra em um fluxo refletido e outro transmitido e, portanto, a Eq. (4) traduz a conservação do fluxo de probabilidade. Nos problemas de espalhamento unidimensionais, as partículas no feixe incidente estão associadas a estados estacionários, em que o momento linear tem um valor bem definido. Nestas circunstâncias é possível mostrar que a densidade de probabilidade é independente do tempo e que a corrente de probabilidade deve permanecer contínua também nos pontos de descontinuidade do potencial $V(x)$. Em termos de funções de onda, isto equivale a ter a função de onda e sua primeira derivada contínuas nestes pontos. Impondo estas condições pode-se mostrar que a condição de unitaridade do espalhamento (4) está intimamente ligada as condições de continuidade da função de onda e de sua primeira derivada impostas nas fronteiras que delimitam as diversas regiões distintas do potencial $V(x)$. Por sua vez, sendo $V(x)$ constante nos domínios dos feixes incidente/refletido e transmitido, então as componentes da função de onda nestas regiões são descritas por

$$
\begin{aligned}
& \varphi_{\mathrm{I}}(x)=A_{\mathrm{I}} e^{i k_{1} x}, \\
& \varphi_{\mathrm{R}}(x)=A_{\mathrm{R}} e^{-i k_{1} x}, \\
& \varphi_{\mathrm{T}}(x)=A_{\mathrm{T}} e^{i k_{3} x},
\end{aligned}
$$

sendo $A_{\mathrm{I}}, A_{\mathrm{R}}$ e $A_{\mathrm{T}}$ suas amplitudes, respectivamente, e sendo $k_{1}$ e $k_{3}$ os números de onda nas regiões do potencial. Portanto, usando as Eqs. (2), (3) e (5) encontraremos que

$$
R(E) \equiv\left|\frac{A_{\mathrm{R}}}{A_{\mathrm{I}}}\right|^{2} ; \quad T(E) \equiv \frac{k_{3}}{k_{1}}\left|\frac{A_{\mathrm{T}}}{A_{\mathrm{I}}}\right|^{2} .
$$

Para calcularmos as razões $A_{\mathrm{R}} / A_{\mathrm{I}}$ e $A_{\mathrm{T}} / A_{\mathrm{I}}$ em termos dos fatores relacionados ao processo de espalhamento, tais como a energia da partícula $E$ e os parâmetros do potencial $V(x)$, é necessário resolvermos a equação de Schrödinger (1) ao longo de todo o domínio do potencial.

Quase a totalidade dos textos básicos de mecânica quântica [11] apresenta as soluções para o problema de espalhamento unidimensional na presença de algum potencial elementar, tal como uma barreira retangular (simétrica e assimétrica), um poço retangular ou um degrau (simples ou duplo) de potencial. Embora todas estas soluções estejam baseadas na resolução da Eq. (1) para a obtenção das probabilidades (6), o desenvolvimento apresentado nos li- 
vros texto passa, talvez, a impressão de que estes potenciais elementares representam diferentes tipos de potenciais e que, portanto, devem ser tratados separadamente. Contudo podemos mostrar que, do ponto de vista prático, na realidade eles não o são. Eles compartilham algumas características comuns que nos permitem concluir que, efetivamente, eles apenas representam diferentes formas de um mesmo potencial geral que engloba em si todos eles como casos particulares. No estudo a seguir nos resolvemos o problema do espalhamento de uma partícula na presença deste potencial geral e obtemos as probabilidades de reflexão e transmissão através dele. Por outro lado, tendo em vista que podemos facilmente controlar o caráter assimétrico deste potencial geral, torna-se muito apropriado e oportuno estudar os efeitos dessa assimetria sobre a transmissão da partícula através dele. Estes são os objetivos deste artigo que está organizado da seguinte maneira: na próxima seção nós apresentamos o potencial unidimensional geral e resolvemos o problema do espalhamento quântico para energias abaixo de sua barreira central, calculando, então, as probabilidades de reflexão e transmissão. $\mathrm{Na}$ seção 3 nós fazemos o mesmo como na seção 2 para energias acima de sua barreira ou poço central. Na seção 4 nós fazemos um estudo numérico dos efeitos da assimetria do potencial sobre a probabilidade de transmissão da partícula. Finalmente, nós fechamos o artigo com alguns comentários breves na seção 5 .

\section{O espalhamento em energias abaixo da barreira central}

Consideremos o espalhamento de partículas quânticas pela seguinte classe de potenciais retangulares unidimensionais:

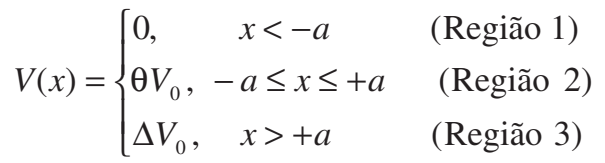

onde a constante positiva $V_{0}$ indica a intensidade do potencial e $2 a$ a sua largura. $O$ parâmetro $\theta$, que determina a natureza do potencial, pode apresentar dois valores possíveis $(\theta= \pm 1)$. Para $\theta=+1$ o potencial (7) apresenta as característica de uma barreira na região 2 enquanto que para $\theta=-1$ ele passa a apresentar as características de um poço de potencial nesta mesma região. O parâmetro de assimetria $\Delta$, cujos valores assumiremos dentro da faixa de $-2 \leq \Delta \leq+2$, determina a simetria $(\Delta=0)$ ou assimetria $(\Delta \neq 0)$ do potencial. Esta faixa de valores para $\Delta$ foi escolhida apenas por conveniência uma vez que os resultados e as conclusões deles advindas são gerais e não mudam para o caso de uma faixa de valores mais ampla. Sendo assim, fica fácil perceber que o potencial (7) pode assumir formas variadas, dependendo dos valores de seus parâmetros. Por exemplo, as formas de uma barreira retangular assimétrica quando $\theta=+1$ e $\Delta=-1$; de um poço simétrico quando $\theta=-1$ e $\Delta=0$; de um degrau duplo quando $\theta=+1$ e $\Delta=+2$, e assim por diante. Na Fig. 1 nós mostramos algumas dessas formas

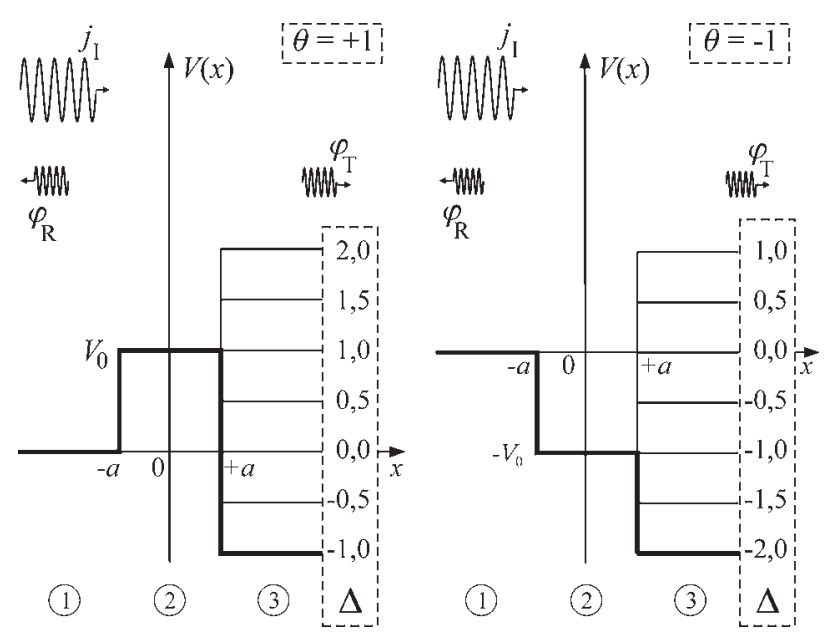

Figura 1 - As diferentes formas que pode tomar o potencial geral (7) para alguns valores particulares dos parâmetros $\theta$ e $\Delta$. Para $\theta=+1$, o potencial geral apresenta as características de uma barreira de potencial $(\Delta<1,0)$, um degrau simples $(\Delta=1,0)$ ou duplo $(\Delta>1,0)$ de potencial. Agora, para $\theta=-1$, estas características são as de um degrau duplo $(\Delta<-1,0)$, simples $(\Delta=-1,0)$ ou de um poço de potencial $(\Delta>-1,0)$.

que pode assumir o potencial geral (7) e os respectivos valores dos parâmetros $\theta$ e $\Delta$ que definem cada uma delas.

Nesta seção nós assumiremos que a energia assintótica $E$ da partícula incidente esteja abaixo da barreira de potencial, ou seja que $E \leq V_{0}$ e que $\theta=+1$. Neste caso, as soluções da equação de Schrödinger independente do tempo em cada uma das três regiões definidas pelo potencial serão dadas por

$$
\begin{array}{ll}
\varphi_{1}(x)=A e^{i k_{1} x}+B e^{-i k_{1} x} & (\text { Região }) \\
\varphi_{2}(x)=C e^{-\kappa_{2} x}+D e^{\kappa_{2} x} & (\text { Região 2) } \\
\varphi_{3}(x)=F e^{i k_{3} x}+G e^{-i k_{3} x} & (\text { Região 3), }
\end{array}
$$

onde $A, B, C, D, F$ e $G$ são coeficientes complexos e

$$
\begin{aligned}
& k_{1}=\frac{\sqrt{2 m E}}{\hbar}, \\
& \kappa_{2}=\frac{\sqrt{2 m\left(V_{0}-E\right)}}{\hbar}, \\
& k_{3}=\frac{\sqrt{2 m\left(E-\Delta V_{0}\right)}}{\hbar},
\end{aligned}
$$

são os números de onda que definem a magnitude do momento em cada região. Por uma questão de clareza e elegância, usaremos em nosso desenvolvimento o, assim denominado, método da matriz de espalhamento [12], que correlaciona a função de onda incidente com aquela emergente, após atravessar o potencial. Dessa forma, considerando o caráter finito de $V(x)$ e aplicando as condições de contorno, $\varphi_{1}(x)=\varphi_{2}(x)$ e $d \varphi_{1}(x) / d x=\varphi_{2}(x) / d x$ em $x=-a$ com as Eqs. (8), encontraremos as relações

$$
\begin{aligned}
& A e^{-i k_{1} a}+B e^{i k_{1} a}=C e^{\kappa_{2} a}+D e^{-\kappa_{2} a} \\
& i k_{1}\left(A e^{-i k_{1} a}-B e^{i k_{1} a}\right)=-\kappa_{2}\left(C e^{\kappa_{2} a}-D e^{-\kappa_{2} a}\right) .
\end{aligned}
$$

Em notação matricial, estas relações podem ser convenientemente escritas como 
$\left(\begin{array}{cc}e^{-i k_{1} a} & e^{i k_{1} a} \\ e^{-i k_{1} a} & -e^{i k_{1} a}\end{array}\right)\left(\begin{array}{l}A \\ B\end{array}\right)=\left(\begin{array}{cc}e^{\kappa_{2} a} & e^{-\kappa_{2} a} \\ \frac{i \kappa_{2}}{k_{1}} e^{\kappa_{2} a} & -\frac{i \kappa_{2}}{k_{1}} e^{-\kappa_{2} a}\end{array}\right)\left(\begin{array}{l}C \\ D\end{array}\right)$.

Segue daí então que,

$\left(\begin{array}{l}A \\ B\end{array}\right)=\frac{1}{2}\left(\begin{array}{cc}e^{i k_{1} a} & e^{i k_{1} a} \\ e^{-i k_{1} a} & -e^{-i k_{1} a}\end{array}\right)\left(\begin{array}{cc}e^{\kappa_{2} a} & e^{-\kappa_{2} a} \\ \frac{i \kappa_{2}}{k_{1}} e^{\kappa_{2} a} & -\frac{i \kappa_{2}}{k_{1}} e^{-\kappa_{2} a}\end{array}\right)\left(\begin{array}{l}C \\ D\end{array}\right)$,

ou

$$
\left(\begin{array}{l}
A \\
B
\end{array}\right)=\mathbf{M}\left(k_{1}, a\right)\left(\begin{array}{l}
C \\
D
\end{array}\right),
$$

onde a matriz $\mathbf{M}\left(k_{1}, a\right)$ é dada por

$\mathbf{M}\left(k_{1}, a\right)=\frac{1}{2}\left(\begin{array}{ll}\left(1+\frac{i \kappa_{2}}{k_{1}}\right) e^{\kappa_{2} a+i k_{1} a} & \left(1-\frac{i \kappa_{2}}{k_{1}}\right) e^{-\kappa_{2} a+i k_{1} a} \\ \left(1-\frac{i \kappa_{2}}{k_{1}}\right) e^{\kappa_{2} a-i k_{1} a} & \left(1+\frac{i \kappa_{2}}{k_{1}}\right) e^{-\kappa_{2} a-i k_{1} a}\end{array}\right)$.

Da mesma forma, aplicando as condições de contorno $\varphi_{2}(x)=\varphi_{3}(x)$ e $d \varphi_{2}(x) / d x=d \varphi_{3}(x) / d x$, em $x=+a$ com as Eqs. (8), obteremos as relações

$$
\begin{aligned}
& F e^{i k_{3} a}+G e^{-i k_{3} a}=C e^{-\kappa_{2} a}+D e^{\kappa_{2} a} \\
& i k_{3}\left(F e^{i k_{3} a}-G e^{-i k_{3} a}\right)=-\kappa_{2}\left(C e^{-\kappa_{2} a}-D e^{\kappa_{2} a}\right),
\end{aligned}
$$

as quais, em notação matricial, ficarão como
$\left(\begin{array}{l}F \\ G\end{array}\right)=\frac{1}{2}\left(\begin{array}{cc}e^{-i k_{3} a} & e^{-i k_{3} a} \\ e^{i k_{3} a} & -e^{i k_{3} a}\end{array}\right)\left(\begin{array}{cc}e^{-\kappa_{2} a} & e^{\kappa_{2} a} \\ \frac{i \kappa_{2}}{k_{3}} e^{-\kappa_{2} a} & -\frac{i \kappa_{2}}{k_{3}} e^{\kappa_{2} a}\end{array}\right)\left(\begin{array}{l}C \\ D\end{array}\right)$.

Tira-se portanto que,

$$
\left(\begin{array}{l}
F \\
G
\end{array}\right)=\mathbf{M}\left(k_{3},-a\right)\left(\begin{array}{l}
C \\
D
\end{array}\right),
$$

onde a matriz $\mathbf{M}\left(k_{3},-a\right)$ será escrita como

$\mathbf{M}\left(k_{3},-a\right)=\frac{1}{2}\left(\begin{array}{ll}\left(1+\frac{i \kappa_{2}}{k_{3}}\right) e^{-\kappa_{2} a-i k_{3} a} & \left(1-\frac{i \kappa_{2}}{k_{3}}\right) e^{\kappa_{2} a-i k_{3} a} \\ \left(1-\frac{i \kappa_{2}}{k_{3}}\right) e^{-\kappa_{2} a+i k_{3} a} & \left(1+\frac{i \kappa_{2}}{k_{3}}\right) e^{\kappa_{2} a+i k_{3} a}\end{array}\right)$.

Usando, agora, as Eqs. (13), (14), (17) e (18) chegamos a relação entre os coeficientes da função de onda em ambos os lados do potencial

$$
\left(\begin{array}{l}
A \\
B
\end{array}\right)=\mathbf{M}\left(k_{1}, a\right) \mathbf{M}^{-1}\left(k_{3},-a\right)\left(\begin{array}{l}
F \\
G
\end{array}\right),
$$

sendo

$\mathbf{M}^{-1}\left(k_{3},-a\right)=\frac{1}{2}\left(\begin{array}{ll}\left(1-\frac{i k_{3}}{\kappa_{2}}\right) e^{\kappa_{2} a+i k_{3} a} & \left(1+\frac{i k_{3}}{\kappa_{2}}\right) e^{\kappa_{2} a-i k_{3} a} \\ \left(1+\frac{i k_{3}}{\kappa_{2}}\right) e^{-\kappa_{2} a+i k_{3} a} & \left(1-\frac{i k_{3}}{\kappa_{2}}\right) e^{-\kappa_{2} a-i k_{3} a}\end{array}\right)$.

Portanto, substituindo as Eqs. (14) e (20) na Eq. (19) encontraremos

$\left.\begin{array}{l}{\left[\alpha_{-} \cosh \left(2 \kappa_{2} a\right)+i \beta_{+} \sinh \left(2 \kappa_{2} a\right)\right] e^{i\left(k_{1}-k_{3}\right) a}} \\ {\left[\alpha_{+} \cosh \left(2 \kappa_{2} a\right)-i \beta_{-} \sinh \left(2 \kappa_{2} a\right)\right] e^{-i\left(k_{1}+k_{3}\right) a}}\end{array}\right)\left(\begin{array}{l}F \\ G\end{array}\right)$,

obtivemos explicitamente a matriz que conecta $A$ e $B \operatorname{com} F$ e $G$ como função de $k_{1} a, \kappa_{2} a$ e $k_{3} a$, essencialmente nós resolvemos todos os problemas sobre o espalhamento quântico por potenciais retangulares unidimensionais, incluindo entre eles potenciais assimétricos, barreiras, poços e degraus de potencial.

Nesta altura, ao supormos que a partícula incidente cruze $V(x)$ vindo da esquerda para a direita (no sentido positivo do eixo $x$ ) obteremos uma solução particular para a equação matricial (21) a qual pode ser estabelecida ao assumirmos $G=0$, no que resulta em ter

$A=\frac{F}{2}\left[\alpha_{+} \cosh \left(2 \kappa_{2} a\right)+i \beta_{-} \sinh \left(2 \kappa_{2} a\right)\right] e^{i\left(k_{1}+k_{3}\right) a}$

$B=\frac{F}{2}\left[\alpha_{-} \cosh \left(2 \kappa_{2} a\right)-i \beta_{+} \sinh \left(2 \kappa_{2} a\right)\right] e^{-i\left(k_{1}-k_{3}\right) a}$

Com as relações acima e as Eqs. (22) encontraremos para as probabilidades de reflexão e transmissão da partícula por $V(x)$ sentado acima constitui o mais elementar baseado na teoria da matriz S. É importante que se ressalte que, uma vez que

$$
R(E)=\left|\frac{B}{A}\right|^{2}=\frac{\left[\left(1-\frac{k_{3}}{k_{1}}\right)^{2} \cosh ^{2}\left(2 \kappa_{2} a\right)+\left(\frac{\kappa_{2}}{k_{1}}+\frac{k_{3}}{\kappa_{2}}\right)^{2} \sinh ^{2}\left(2 \kappa_{2} a\right)\right]}{\left[\left(1+\frac{k_{3}}{k_{1}}\right)^{2} \cosh ^{2}\left(2 \kappa_{2} a\right)+\left(\frac{\kappa_{2}}{k_{1}}-\frac{k_{3}}{\kappa_{2}}\right)^{2} \sinh ^{2}\left(2 \kappa_{2} a\right)\right]},
$$




$$
T(E)=\frac{k_{3}}{k_{1}}\left|\frac{F}{A}\right|^{2}=\frac{4 k_{3} / k_{1}}{\left[\left(1+\frac{k_{3}}{k_{1}}\right)^{2} \cosh ^{2}\left(2 \kappa_{2} a\right)+\left(\frac{\kappa_{2}}{k_{1}}-\frac{k_{3}}{\kappa_{2}}\right)^{2} \sinh ^{2}\left(2 \kappa_{2} a\right)\right]} .
$$

Analisando estes resultados gerais podemos identificar algumas propriedades interessantes sobre o espalhamento do sistema.

- Para $E<V(x)$ e $\Delta=+1$, que representa um degrau de potencial localizado em $x=-a$, teremos $k_{3}=\sqrt{2 m\left(E-V_{0}\right)} / \hbar$ $=\mathrm{i} \kappa_{2}$ e pelas Eqs. (23) e (24) encontraremos

$$
\frac{B}{A}=\frac{\left(1-i \kappa_{2} / k_{1}\right) e^{-i k_{1} a}}{\left(1+i \kappa_{2} / k_{1}\right) e^{i k_{1} a}}
$$

uma vez que a largura de tal potencial será dada pelo limite $2 a \rightarrow \infty$. Com este resultado pode-se mostrar que $R=1$; isto é, teremos uma reflexão total da onda incidente. Na região interna ao degrau de potencial teremos uma onda evanescente (exponencialmente atenuada) que desaparece gradualmente ao penetrar no degrau.

- Para $\Delta=0$ teremos $k_{3}=k_{1}=\sqrt{2 m E} / \hbar$, o que corresponde a uma barreira de potencial retangular simétrica. Neste caso a Eq. (26) nos fornece

$$
T(E)=\frac{1}{1+\left[1+\frac{1}{4}\left(\frac{\kappa_{2}}{k_{1}}-\frac{k_{1}}{\kappa_{2}}\right)^{2}\right] \sinh ^{2}\left(2 \kappa_{2} a\right)},
$$

um resultado bem conhecido o qual é denominado de fator de tunelamento.

\section{O espalhamento em energias acima da barreira ou poço central}

Nesta seção consideraremos que a partícula incidente possui energia assintótica $E$ maior que $\theta V_{0}$ ou $\Delta V_{0}$, onde agora $\theta$ pode assumir qualquer um dos dois valores +1 ou
-1. Sendo assim, a solução da equação de Schrödinger na região 2 deverá também ser oscilatória, como nas outras duas regiões. Portanto, podemos nos utilizar de um processo de continuação analítica e substituir $\kappa_{2}$ por $i k_{2}$ de modo a obter

$$
\begin{aligned}
& k_{1}=\frac{\sqrt{2 m E}}{\hbar}, \\
& k_{2}=\frac{\sqrt{2 m\left(E-\theta V_{0}\right)}}{\hbar}, \\
& k_{3}=\frac{\sqrt{2 m\left(E-\Delta V_{0}\right)}}{\hbar},
\end{aligned}
$$

para os novos números de onda nas três regiões. Com este procedimento é possível reescrever a função de onda na região interna ao potencial como

$$
\varphi_{2}(x)=C e^{-i k_{2} x}+D e^{i k_{2} x} \quad(\text { Região } 2)
$$

enquanto que $\varphi_{1}(x)$ e $\varphi_{3}(x)$ mantém-se dadas pelas mesmas expressões apresentadas pelas Eqs. (8). Como no caso da seção anterior, consideraremos que a incidência da partícula sobre $V(x)$ seja no sentido positivo do eixo $x$ e, com isso, as Eqs. (23) e (24) podem agora ser escritas como

$A=\frac{F}{2}\left[\alpha_{+} \cos \left(2 k_{2} a\right)-i \beta_{+} \sin \left(2 k_{2} a\right)\right] e^{i\left(k_{1}+k_{3}\right) a}$

$B=\frac{F}{2}\left[\alpha_{-} \cos \left(2 k_{2} a\right)+i \beta_{-} \sin \left(2 k_{2} a\right)\right] e^{-i\left(k_{1}-k_{3}\right) a}$

Portanto, para espalhamentos com energias acima da barreira ou poço central do potencial obteremos para as probabilidades de reflexão e transmissão da partícula por $V(x)$

$$
\begin{aligned}
& R(E)=\left|\frac{B}{A}\right|^{2}=\frac{\left[\left(1-\frac{k_{3}}{k_{1}}\right)^{2} \cos ^{2}\left(2 k_{2} a\right)+\left(\frac{k_{2}}{k_{1}}-\frac{k_{3}}{k_{2}}\right)^{2} \sin ^{2}\left(2 k_{2} a\right)\right]}{\left[\left(1+\frac{k_{3}}{k_{1}}\right)^{2} \cos ^{2}\left(2 k_{2} a\right)+\left(\frac{k_{2}}{k_{1}}+\frac{k_{3}}{k_{2}}\right)^{2} \sin ^{2}\left(2 k_{2} a\right)\right]}, \\
& T(E)=\frac{k_{3}}{k_{1}}\left|\frac{F}{A}\right|^{2}=\frac{4 k_{3} / k_{1}}{\left[\left(1+\frac{k_{3}}{k_{1}}\right)^{2} \cos ^{2}\left(2 k_{2} a\right)+\left(\frac{k_{2}}{k_{1}}+\frac{k_{3}}{k_{2}}\right)^{2} \sin ^{2}\left(2 k_{2} a\right)\right]} .
\end{aligned}
$$

Com os resultados encontrados acima podemos destacar alguns aspectos importantes.

- Para $\theta=+1$ e $\Delta=0$, teremos $k_{1}=k_{3}=\sqrt{2 m E} / \hbar, k_{2}=$ $\sqrt{2 m\left(E-V_{0}\right)} / \hbar$ e $T(E)$ se reduz a

$$
T(E)=\frac{1}{1+\frac{1}{4}\left(\frac{k_{2}}{k_{1}}-\frac{k_{1}}{k_{2}}\right)^{2} \sin ^{2}\left(2 k_{2} a\right)},
$$

que corresponde ao resultado usual para o caso de uma barreira de potencial retangular simétrica.

- Para $\theta=-1$ e $\Delta=0$, teremos $k_{1}=k_{3}=\sqrt{2 m E} / \hbar, k_{2}=$ $\sqrt{2 m\left(E+V_{0}\right)} / \hbar$ e encontraremos a mesma expressão mostrada acima para a transmissão $T(E)$ através de $V(x)$, que agora, corresponde a um poço retangular simétrico de potencial.

- Para $\theta=+1$ e $\Delta=+1$, teremos $k_{1}=\sqrt{2 m E} / \hbar, k_{2}=k_{3}=$ $\sqrt{2 m\left(E-V_{0}\right)} / \hbar$, e encontraremos 


$$
T(E)=\frac{4 k_{3} / k_{1}}{\left(1+k_{3} / k_{1}\right)^{2}},
$$

que é o resultado já bem conhecido para a probabilidade de transmissão através de um degrau de potencial $V_{0}$ com $E>V_{0}$.

- Para $\theta=+1$ e $\Delta=+2$, teremos $k_{1}=\sqrt{2 m E} / \hbar, k_{2}=$ $\sqrt{2 m\left(E-V_{0}\right)} / \hbar$ e $k_{3}=\sqrt{2 m\left(E-2 V_{0}\right)} / \hbar$. Portanto $k_{1}>k_{2}>k_{3}$ e pela Eq. (32) encontraremos

$$
T(E)=\frac{4 k_{1} k_{3} k_{2}^{2}}{k_{2}^{2}\left(k_{1}+k_{3}\right)^{2}+\left(k_{3}^{2}-k_{2}^{2}\right)\left(k_{1}^{2}-k_{2}^{2}\right) \sin ^{2}\left(2 k_{2} a\right)},
$$

para a transmissão através de um degrau duplo de potencial com $E>2 V_{0}$.

\section{Potenciais assimétricos e a probabilidade de transmissão}

Nesta seção apresentaremos os resultados de um estudo numérico dos efeitos da assimetria do potencial sobre a probabilidade de transmissão através dele. Primeiro consideraremos o caso da incidência de elétrons sobre um potencial $V(x)$ que apresenta as característica de uma barreira retangular assimétrica de potencial $(\theta=+1)$, onde tomare- mos $-1,0 \leq \Delta \leq+1,0$. Assumiremos que esta barreira possui altura $V_{0}=10 \mathrm{eV}$, da ordem do dôbro da energia de Fermi dos elétrons em um metal, e largura $2 a=20 \AA$, que corresponde a um sistema com dimensões próximas a umas poucas camadas atômicas de óxido separando duas lâminas de metal, como por exemplo o caso de $\mathrm{Ni}-\mathrm{NiO}-\mathrm{Pb}$.

A Fig. 2(A) mostra a probabilidade de transmissão $T$, obtida com as Eqs. (26) e (32), como função de $E / V_{0}$ e $\Delta$. Podemos observar as ressonâncias características de $T$, e $T \rightarrow 1$ com o aumento da energia da partícula incidente. De modo a ter uma visualização mais clara da dependência de $T$ com $\Delta$ (e, dessa forma com o caracter assimétrico do potencial), mostramos na Fig. 2(B) três curvas resultantes dos cortes na Fig. 2(A) para os valores de $\Delta=-0,5 ; 0,0$; $+0,5$. Em geral, para uma dada energia acima da barreira, observamos um aumento na transmissão conforme os valores de $\Delta$ também aumentam. Isto é, verifica-se mais transmissão através da barreira conforme o potencial na região 3 é mais alto que na região 1 . Isto fica mais claramente perceptível no comportamento de $T$ para pontos nas vizinhanças dos mínimos mostrados na Fig. 2(B). Contudo, como pode ser visto na Fig. 2(C), este comportamento é ligeiramente invertido nas ressonâncias (máximos) de $T$. Esta figura apresenta $T$ como função de $\Delta$ para os três máximos da Fig. 2(B), indicados como $T\left(E_{n}, \Delta\right) ; \mathrm{n}=1,2$ e 3.
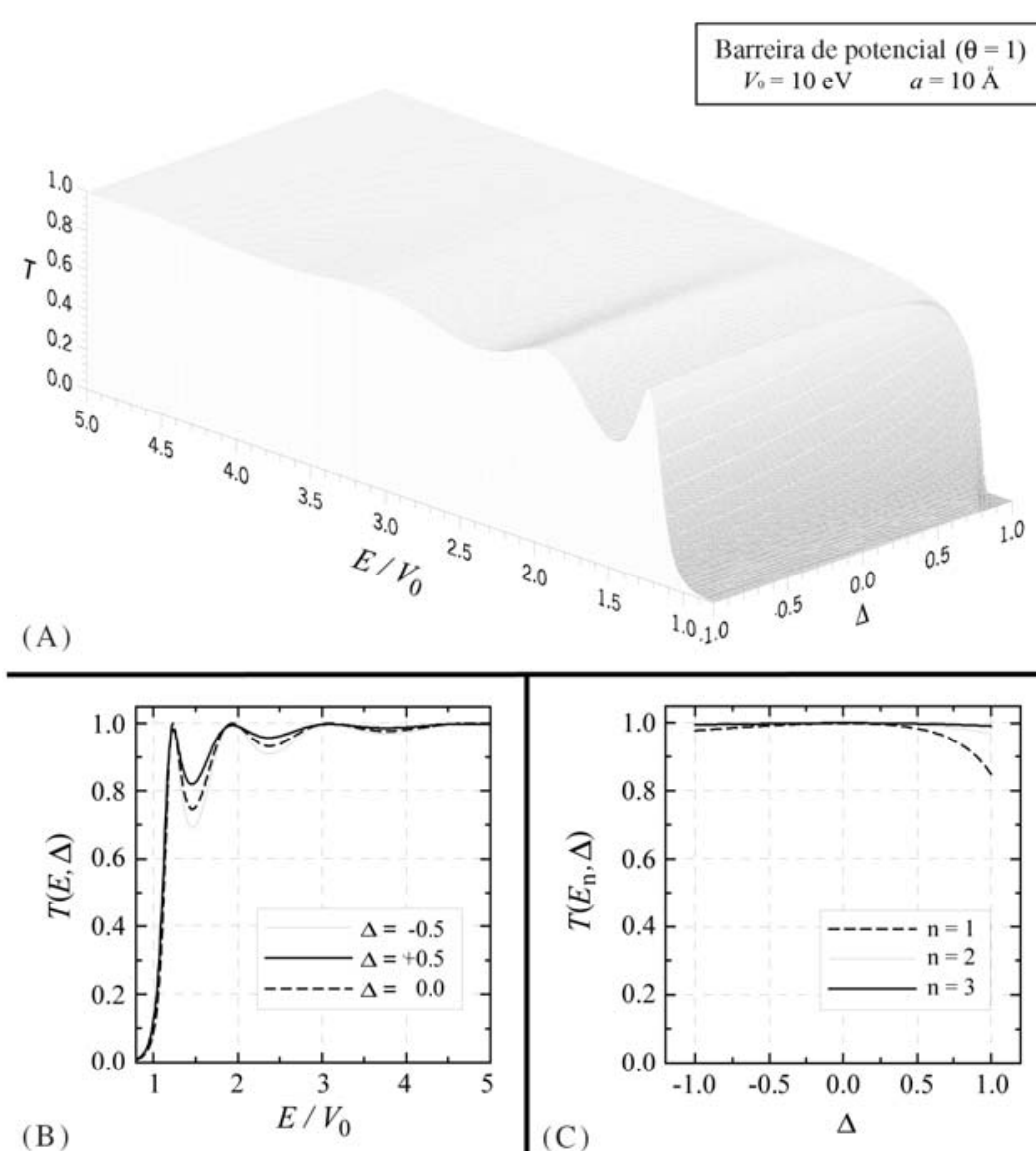

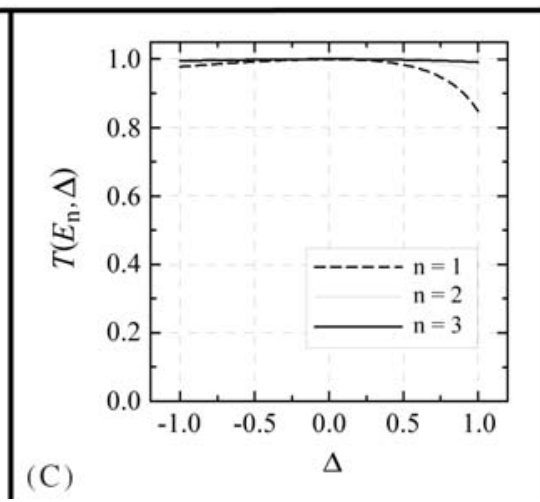

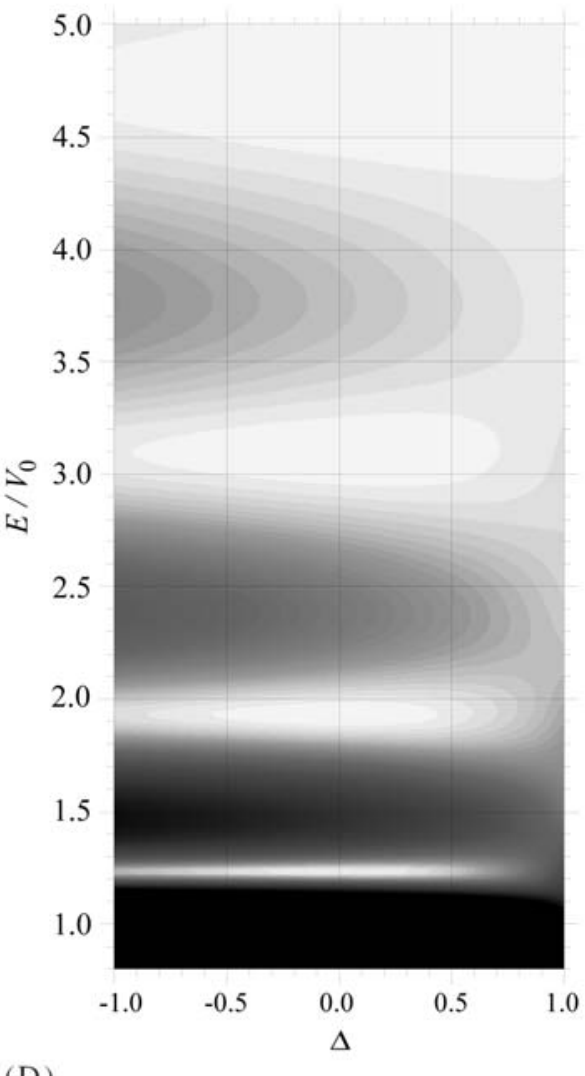

(D)

Figura 2 - (A) A probabilidade de transmissão $T$ como uma função de $E / V_{0}$ e $\Delta$ para o espalhamento de um elétron por uma barreira de potencial com $\theta=+1, V_{0}=10 \mathrm{eV}, a=10 \AA \mathrm{e}-1,0 \leq \Delta \leq+1,0$. (B) $T$ como uma função de $E / V_{0}$ para três valores do parâmetro de assimetria, $\Delta=-0,5 ; 0,0 ;+0,5$. (C) $T$ como uma função de $\Delta$ para três energias de ressonância com $n=1,2$ e 3. (D) A visualização por cima da figura (A). Aqui quanto mais escura é a região sombreada, mais próxima de zero é $T$. 
Neste caso podemos observar que, para um dado máximo localizado em $E_{n}$, a transmissão para valores positivos de $\Delta$ é menor do que para os valores negativos deste parâmetro. Além disso, para cada uma dessas curvas de máximos da transmissão, o maior valor para $T\left(E_{n}, \Delta\right)$ acontece quando o potencial é simétrico $(\Delta=0)$. Estes valores característicos $E_{n}$ dos máximos de $T(E)$ podem ser determinados através da condição

$$
2 k_{2} a=n \pi, \quad n=1,2,3,
$$

imposta a Eq. (32), e que, do ponto de vista ondulatório, traduz a condição necessária para que se obtenha uma interferência construtiva entre as ondas incidente e refletida na região interna à barreira de potencial (região 2). Do ponto de vista da ótica esta condição, normalmente utilizada para minimizar a quantidade de luz refletida por instrumentos óticos, nada mais é do que aquela que descreve o interfermetro de Fabry-Perot. Agora, do ponto de vista de energia, a condição acima equivale a ter

$$
E_{n}=n^{2} \frac{\hbar^{2} \pi^{2}}{8 m a^{2}}+V_{0}
$$

Os picos mais estreitos de $T\left(E_{n}, \Delta\right)$, observados para as energias mais baixas, são denominados de ressonâncias de transmissão e as energias $E_{n}$ que definem as suas posições são justamente aquelas correspondentes aos estados ligados de um poço infinito de igual largura. O razão disto está no fato dos dois fenômenos compartilharem a mesma condição (33). Este fenômeno de ressonância de transmissão tanto é observado em física atômica, no espalhamento de elétrons de baixa energia por átomos de gases nobres (Ar, $\mathrm{Ne}$ ) quanto em física nuclear, no espalhamento de neutrons com energias de uns poucos $\mathrm{MeV}$ por núcleos. No caso da física atômica o fenômeno recebe o nome de efeito Ramsauer-Townsend [13] enquanto que na física nuclear ele é conhecido como ressonâncias de tamanho ou estados de partícula única. Outra coisa a destacar na Fig. 2(C) é o fato da dependência de $T$ com $\Delta$ ficar menos acentuada para valores maiores de $n$. A Fig. 2(D) mostra a visualização por cima da Fig. 2(A). Nesta figura conforme o sombreamento se torna mais escuro, mais próxima de zero fica a probabilidade de transmissão. Conforme já tínhamos concluído antes, para um dado valor da energia acima do topo da barreira $\left(E / V_{0}>1\right)$, a transmissão através da barreira é reduzida no caso de valores negativos de $\Delta$. Este comportamento se inverte nas ressonâncias, isto é, nos máximos de $T$ (mostrados pelas regiões mais claras na Fig. 2(D)). Todo este complexo comportamento de $T$ em termos de $E$ e $\Delta$ é resultado da interferência entre as ondas incidentes e refletidas nos pontos onde o potencial apresenta variações bruscas.

Os resultados mostrados na Fig. 3 correspondem aqueles apresentandos na Fig. 2 só que para um potencial com a largura aumentada para $2 a=40 \AA$ A. Como podemos observar, todos os resultados em geral são descritos como no caso anterior, tendo-se apenas um aumento no número de ressonâncias em $T$. Uma maior proporção entre a largura da barreira (2a) e o comprimento de onda na região 2 é responsável por um número maior de ressonâncias em $T$.

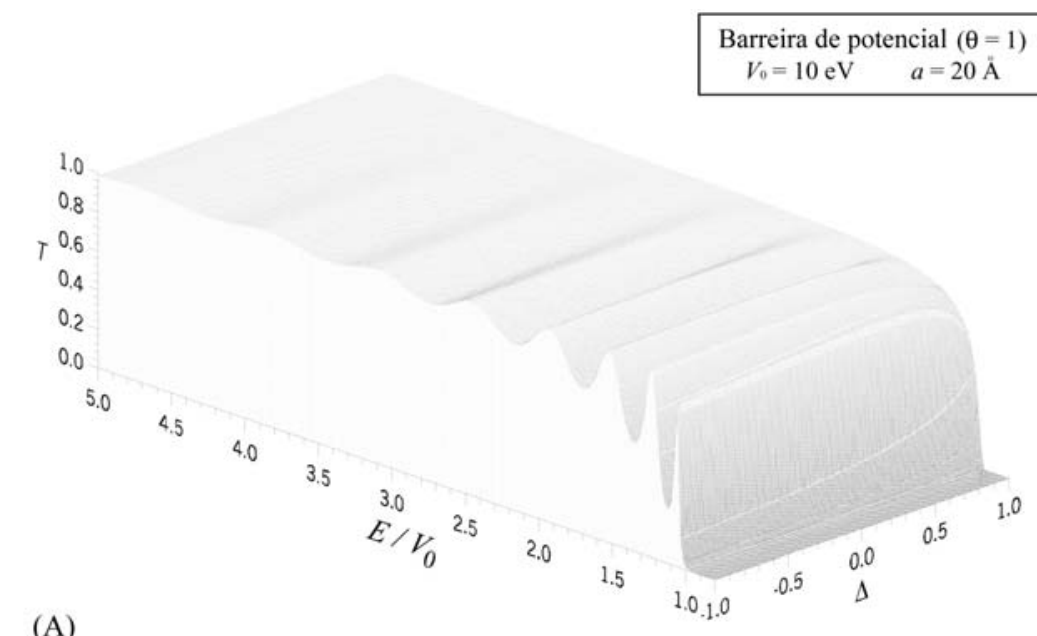

(A)

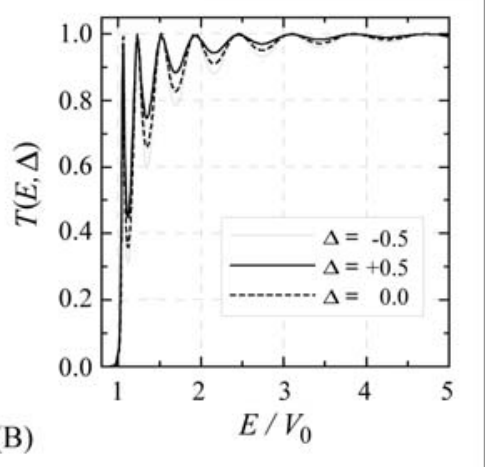

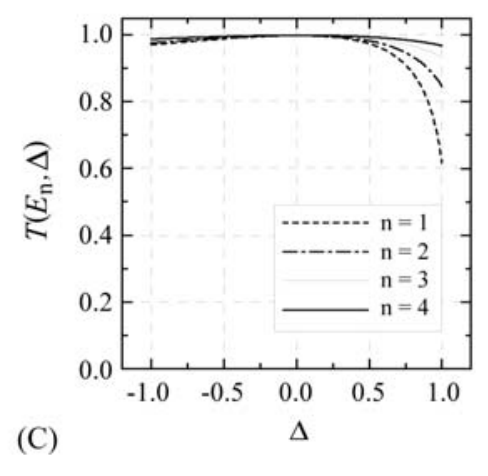

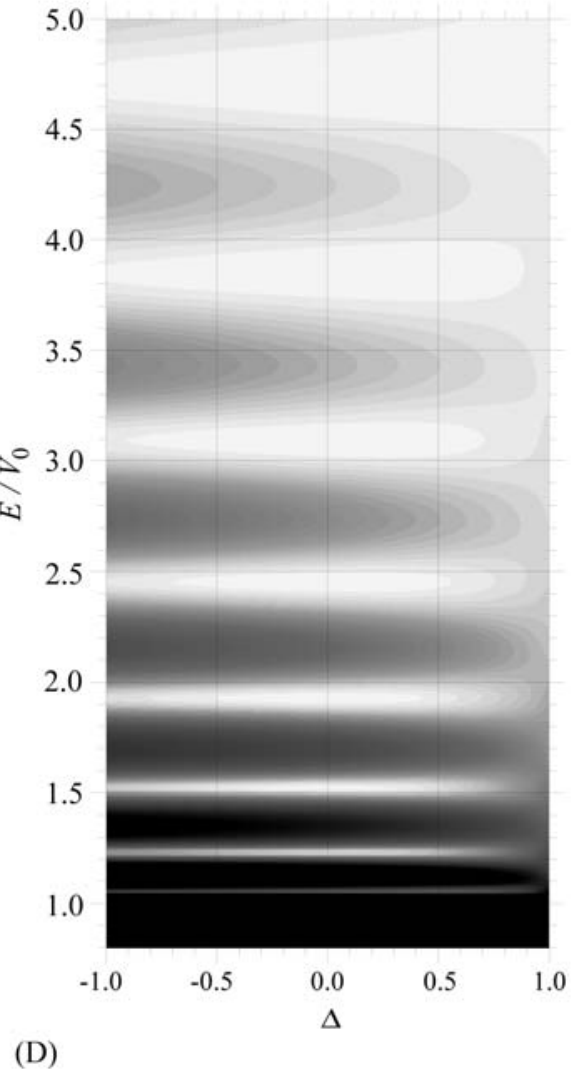

Figura 3 - O mesmo mostrado na Fig. 2, só que para o caso da largura do potencial ser $a=20 \AA$ e $n=1,2,3$ e 4. 
A Fig. 4 apresenta os resultados obtidos para o caso de um elétron incidente sobre um potencial $V(x)$ que apresenta as característica de um poço retangular de potencial $(\theta=-1)$. Os valores de todos os outros parâmetros são mantidos iguais aqueles usados para os resultados mostrados na Fig. 2, isto é, $-1,0 \leq \Delta \leq+1,0$, sendo a profundidade e a largura do poço de potencial dadas, respectivamente, por $V_{0}=10$ eV e $2 a=20 \AA$. Novamente, $T$ é mostrado como uma função de $E / V_{0}$ e $\Delta$ (Fig. 4(A)) e também apresenta ressonâncias. Contudo, diferente do caso de uma barreira de potencial, agora, no caso de um poço de potencial, para uma dada energia nós verificamos um aumento da transmissão para valores negativos de $\Delta$, isto é, quando o potencial na região 3 é mais baixo que na região 1 (Fig. 4(B)). Este aumento em $T$ mostra-se especialmente proeminente para energias $E / V_{0}<1$. Uma outra coisa a destacar é que neste caso o comportamento geral de $T$ em termos de $\Delta$ não é invertido nas ressonâncias (Fig. 4(C)). Para este caso do espalhamento de um elétron por um poço de potencial as energias de ressonâncias na transmissão são dadas por

$$
E_{n}=n^{2} \frac{\hbar^{2} \pi^{2}}{8 m a^{2}}-V_{0}
$$

sendo $n$ grande o bastante para termos $E_{n}>0$. Novamente, como para barreira de potencial, o maior valor para $T\left(E_{n}, \Delta\right)$ acontece quando o poço é simétrico $(\Delta=0)$. Uma visua- lização por cima da Fig. 4(A) é mostrada na Fig. 4(D). Ao contrário do caso anterior (Figs. 2(D) e 3(D)), podemos verificar que agora a região mais escura (menores valores para $T$ ) está concentrada à direita na Fig. 4(D), indicando assim a redução na transmissão do elétron como resultado do aumento da altura da lateral do poço de potencial ligada ao parâmetro de assimetria $\Delta$.

Todas as conclusões descritas acima para o espalhamento de um elétron por um poço de potencial permanecem quando a sua largura é aumentada para $2 a=40 \AA$ (Fig. 5); apenas observamos um número maior de ressonâncias em $T$, cuja explicação é aquela mesma citada para o caso da barreira.

Após termos apresentado os resultados numéricos para a probabilidade de transmissão no potencial $V(x)$ quando ele apresenta as característica de uma barreira e de um poço de potencial podemos comparar estes resultados e observar que eles possuem uma superfície de fronteira comum. A curva obtida pelo corte na superfície apresentada na Fig. 2(A) para $T(E)$ de uma barreira de potencial quando $\Delta=1$ é a mesma que obteríamos se fizéssemos um corte na superfície apresentada na Fig. 4(A) para $T(E)$ de um poço de potencial quando $\Delta=-1$. A única diferença entre as duas curvas seria o deslocamento em energia de $\Delta E=10 \mathrm{eV}$ originado pela apresentação da energia de colisão em cada uma das duas situações. Isto pode ser melhor visualizado se compararmos as Figs. 2(D) e 4(D).
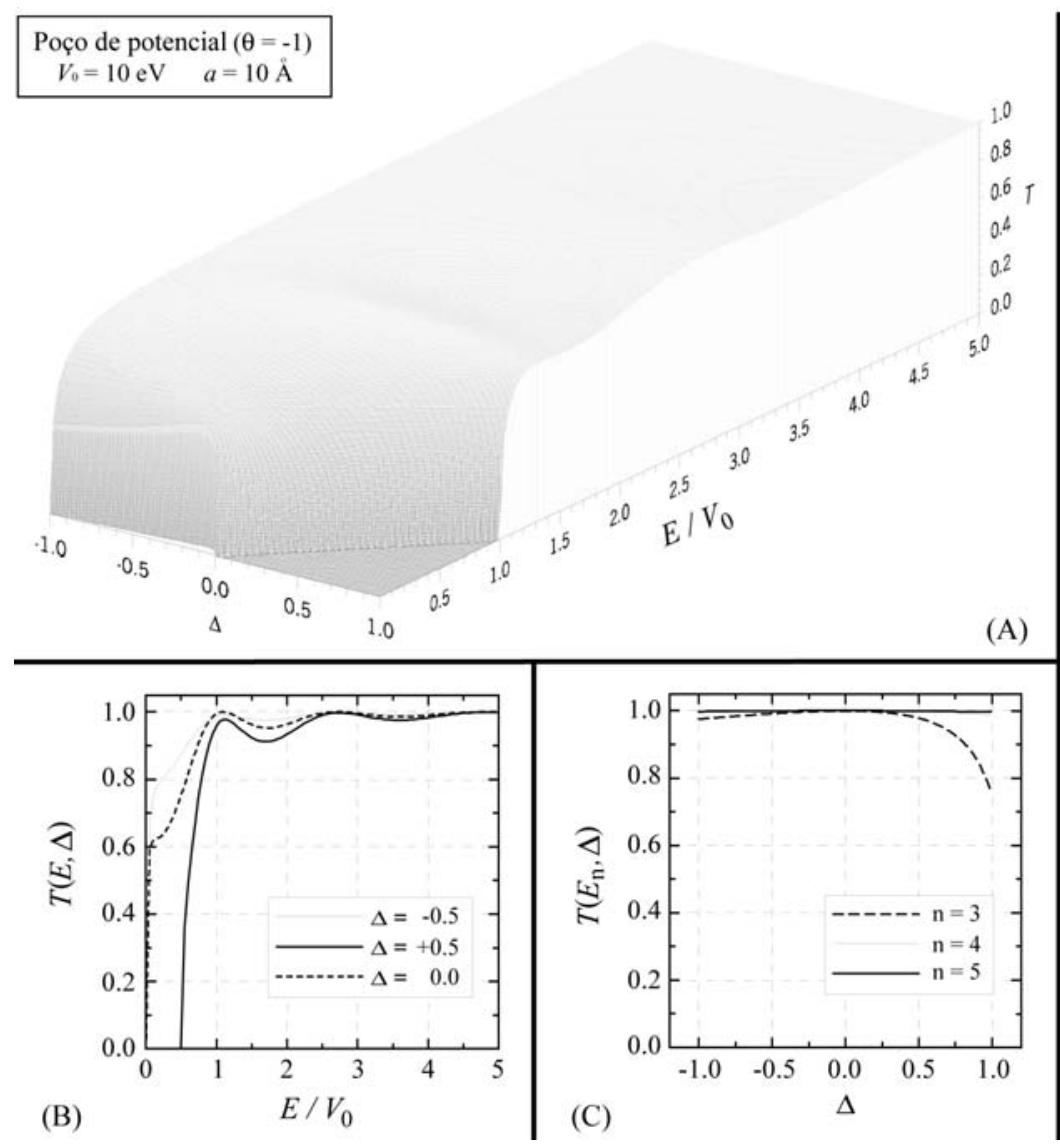

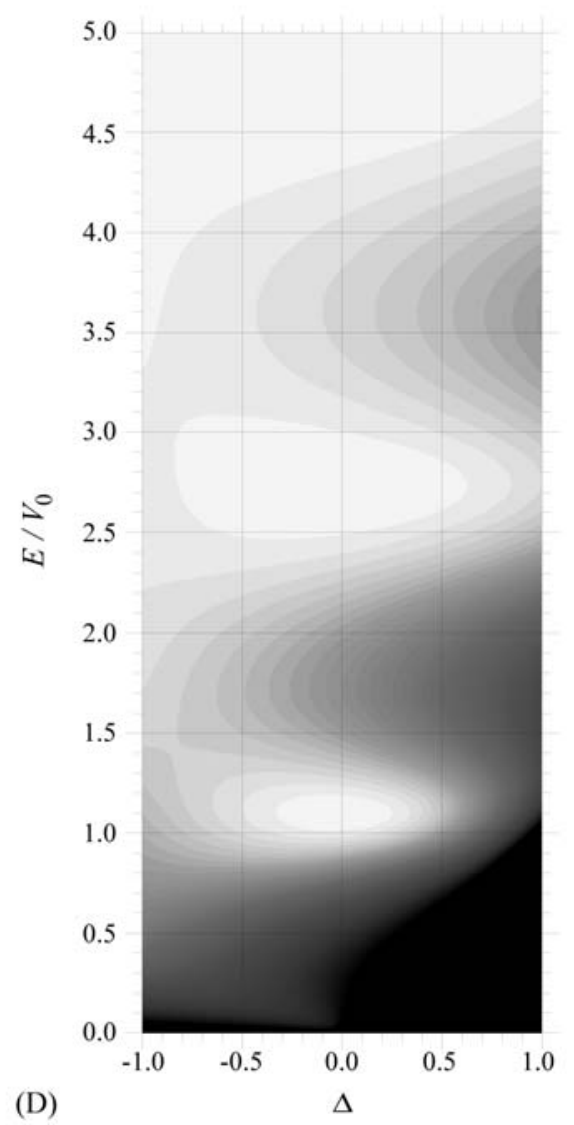

Figura 4 - A) A probabilidade de transmissão $T$ como uma função de $E / V_{0}$ e $\Delta$ para o espalhamento de um elétron por um poço de potencial com $\theta=-1$, $V_{0}=10 \mathrm{eV}, a=10 \AA ̊ \mathrm{e}-1,0 \leq \Delta \leq+1,0$. (B) $T$ como uma função de $E / V_{0}$ para três valores do parâmetro de assimetria, $\Delta=-0,5 ; 0,0 ;+0,5$. (C) $T$ como uma função de $\Delta$ para três energias de ressonância com $n=3,4$ e 5. (D) A visualização por cima da figura (A). Novamente, quanto mais escura é a região sombreada, mais próxima de zero é $T$. 


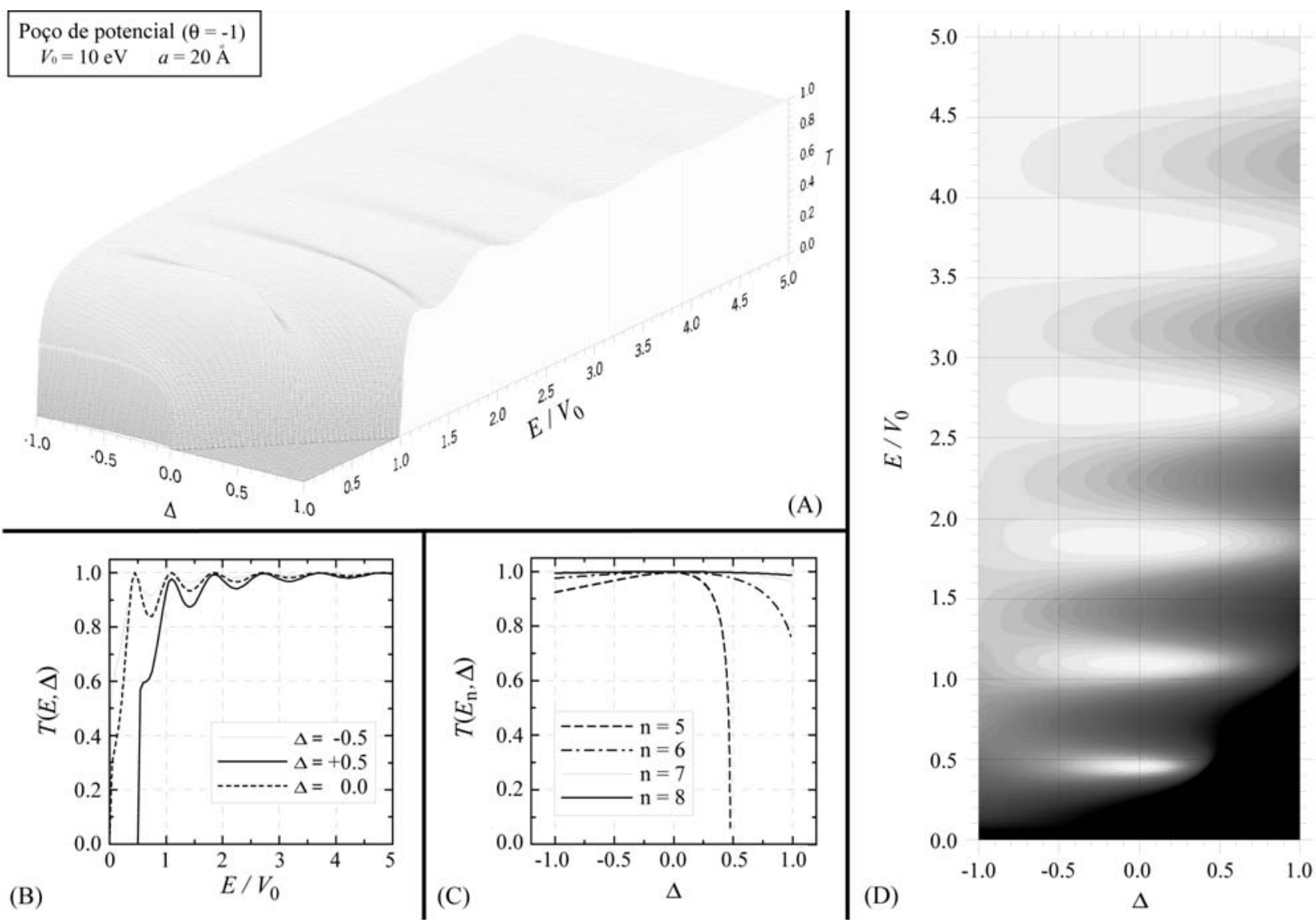

Figura 5 - O mesmo mostrado na Fig. 2, só que para o caso da largura do potencial ser $a=20 \AA$ e $n=5,6,7$ e 8 .

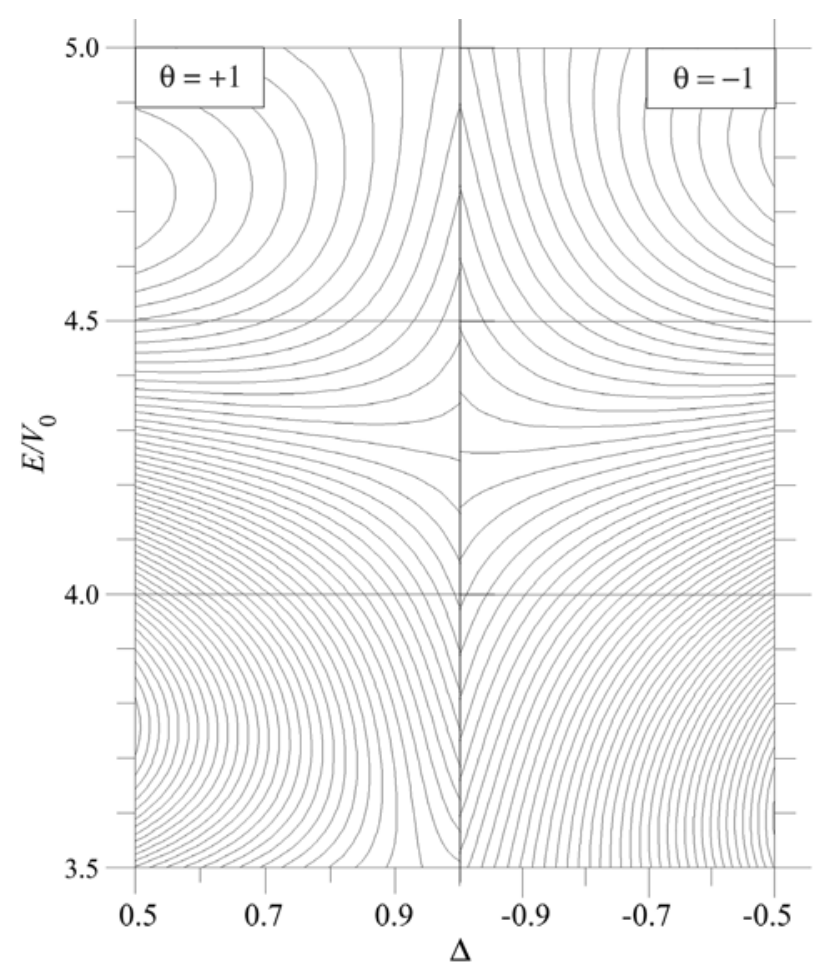

Figura 6 - As curvas de nível projetadas no plano $E / V_{0} \times \Delta$ para a probabilidade de transmissão $T$ de um elétron por um poço de potencial $(\theta=-1)$ e uma barreira de potencial $(\theta=+1)$ no caso em que $V_{0}=10 \mathrm{eV}$.

De modo a tornar mais clara esta comparação apresentamos na Fig. 6 as curvas de nível projetadas no plano $E / V_{0} \times \Delta$ para a probabilidade de transmissão obtidas para o poço de potencial $(\theta=-1)$ e para a barreira de potencial $(\theta=+1)$, unidas pelos lados $\Delta=-1$, no caso do poço, com $\Delta=+1$, no caso da barreira. Como mostra a figura, estas curvas nos dois lados tendem a um valor comum na linha que une as duas figuras. Algumas pequenas diferenças observadas entre elas nesta linha de separação são ocasionadas pelas limitações numéricas do programa usado para obtê-las. A explicação para esta fronteira em comum entre as duas formas de características distintas para $V(x)$ está mostrada na Fig. 1. Para estes valores dos parâmetros $\theta$ e $\Delta$ nas duas situações o potencial $V(x)$ resultante é o mesmo: um degrau de potencial de altura $V_{0}$.

Uma observação final a ser feita é que, ao contrário das seções anteriores, não desenvolvemos também nesta seção um estudo da probabilidade de reflexão no potencial geral $V(x)$ tendo em vista que ele não seria necessário. A condição da conservação da probabilidade de encontrar a partícula, traduzida pela Eq. (4), permite obter imediatamente $R(E)$ a partir do conhecimento da probabilidade de transmissão $T(E)$. Dessa forma, como $R(E)=1-T(E)$, então todas as características relacionadas com o estudo numérico/gráfico de $T(E)$ podem ser direcionadas ao seu complemento à unidade e assim aplicadas para $R(E)$.

\section{Conclusões}

Neste estudo nós apresentamos um potencial geral que engloba todos os potencials retangulares unidimen- 
sionais elementares, tais como: barreiras, poços e degraus de potencial. Nós usamos este potencial geral para estudar o espalhamento de uma partícula quântica e obtivemos as suas probabilidades de reflexão $R$ e transmissão $T$ através dele. Mostramos que com uma escolha conveniente dos parâmetros deste potencial geral nossos resultados podem fornecer as expressões bem conhecidas para $T$ e $R$ para cada forma particular englobada por este potencial geral. Tendo em vista o fácil contrôle sobre o caráter assimétrico do potencial geral em nossos resultados, fizemos um estudo numérico dos efeitos da assimetria do potencial na transmissão da partícula. Nossas conclusões neste caso são que no espalhamento de um elétron por uma barreira assimétrica, em geral, para uma dada energia acontece um aumento na transmissão conforme o parâmetro de assimetria que caracteriza o potencial aumenta. Este comportamento da transmissão é invertido nas ressonâncias de $T$. No caso do espalhamento de um elétron por um poço assimétrico de potencial, ao contrário do caso da barreira, observamos um aumento na transmissão para todas as energias conforme o parâmetro de assimetria do poço diminui. Tanto para a barreira como para o poço de potencial, este comportamento da transmissão pode ser visto como uma manifestação da natureza ondulatória do sistema.

Este estudo dos efeitos da assimetria do potencial sobre a transmissão de uma partícula através dele pode ter aplicações interessantes nas áreas das físicas molecular, atômica e nuclear. Como de fato, poderíamos simular os efeitos de canais acoplados em física nuclear usando uma barreira de potencial assimétrica [14] e dessa forma estudar, através de um modelo teórico muito simples, o espalhamento na presença de graus de liberdade internos. Como uma outra aplicação, os nossos resultados poderiam também ser usados como um guia para uma melhor compreensão das principais características presentes nos processos de tunelamento de partículas ligadas [15]. Poços duplos de potenciais assimétricos podem ser usados para modelar tais sistemas cujo principal aspecto quântico está relacionado à indisponibilidade de todas as energias das partículas. Contudo, a região interna de um potencial quadrado assimétrico com duplo mínimo poderia ser simulado por uma barreira retangular assimétrica. Tal estudo teria, por exemplo, aplicações muito interessantes em biofísica ao ser usado como uma modelagem simples para proteínas. Nossos resultados também poderiam ser usados na física do estado sólido como um estudo introdutório sobre o tunelamento em semi-condutores [16] uma vez que estes dispositivos, então obtidos pela dopagem de certos materiais, poderiam também ser simulados por potenciais assimétricos. Neste sentido o trabalho desenvolvido por B. Ricco e M. Ya Azbel [17] sobre o tunelamento através dos estados do poço criado por uma barreira dupla e unidimensional de potencial (tunelamento ressonante) pode ser visto como um degrau de ligação entre o estudo simples e didático que apresen- tamos neste trabalho e estudos mais avançados direcionados para esta área da física. No trabalho citado acima os autores fazem uma elegante discussão dos fenômenos interessantes relacionados ao tunelamento quântico através de uma barreira dupla de potencial (tais como captura de portadores de carga, efeitos da simetria da barreira na ressonância do tunelamento, tempo requerido para o completo estabelecimento da ressonância de tunelamento, efeitos da temperatura na corrente produzida, condições experimentais requeridas para a otimização da ressonância de tunelamento através de estruturas com eletrodos semicondutores) quando é considerada a ação de um campo elétrico externo.

\section{Agradecimentos}

M.A.C.R. agradece ao suporte financeiro dado pela Fundação de Amparo à Pesquisa do Estado de São Paulo FAPESP (contrato no. 98/13722-2). Os autores também gostariam de agradecer ao professor Diógenes Galetti do Instituto de Física Teórica - UNESP pela leitura crítica e pelas valiosas sugestões feitas.

\section{Referências}

[1] Veja, por exemplo, (a) H. Enge, M.R. Wehr and J.A. Richards, Introduction to Atomic Physics (Addison-Wesley, Menlo Park, 1972); (b) K. Heyde, Basic Ideas and Concepts in Nuclear Physics (Institute of Physics Publishing, Bristol, 1994).

[2] G. Gamow, Z. Physik 51, 204 (1928); Z. Physik 52, 510 (1928).

[3] R.W. Gurney and E.U. Condon, Nature 122, 439 (1928); Phys. Rev. 33, 127 (1929).

[4] R.A. Millikan and C.F. Eyring. Phys. Rev. 27, 51 (1926).

[5] C.C. Tannoudji, B. Diu and F. Laloë, Quantum Mechanics (John Wiley \& Sons, New York, 1977), v. 1.

[6] R. Tsu and L. Esaki, App. Phys. Lett. 22, 562 (1973).

[7] C.W. J. Beenakker and H. van Houten, in Quantum Transport in Semicondutor Nanostructures, edited by H. Turnbull (Academic Press, San Diego, 1991), Solid State Physics, v. 44.

[8] C.W.J. Beenakker and A.A.M. Staring, Phys. Rev. B 46, 9667 (1992).

[9] N. Maitra and E.J. Heller, Phys. Rev. Lett. 78, 3035 (1997).

[10] C.V. Reddy, V. Narayanamurti, J.H. Ryou, U. Chowdhury and R.D. Dupuis, App. Phys. Lett. 77, 1167 (2000).

[11] Veja, por exemplo, (a) L. Landau and E. Lifshitz, Quantum Mechanics (Pergamom, New York, 1977); (b) R.L. Liboff, Introductory Quantum Mechanics (Addison-Wesley, Menlo Park, 1992); (c) F. Schwabl, Quantum Mechanics (Springer, Berlin, 1995).

[12] E. Merzbacher, Quantum Mechanics (John Wiley \& Sons, New York, 1961).

[13] C.W. Ramsauer, Ann. Phys. 64, 513 (1921).

[14] A.B. Balantekin and N. Takigawa, Rev. Mod. Phys. 70, 77 (1998).

[15] M.D. Harmony, Chem. Soc. Rev. 1, 211 (1972).

[16] C. Kittel, Introduction to Solid State Physics (John Wiley \& Sons, New York, 1971).

[17] B. Ricco and M. Ya Azbel, Phys. Rev. B 29, 1970 (1984). 